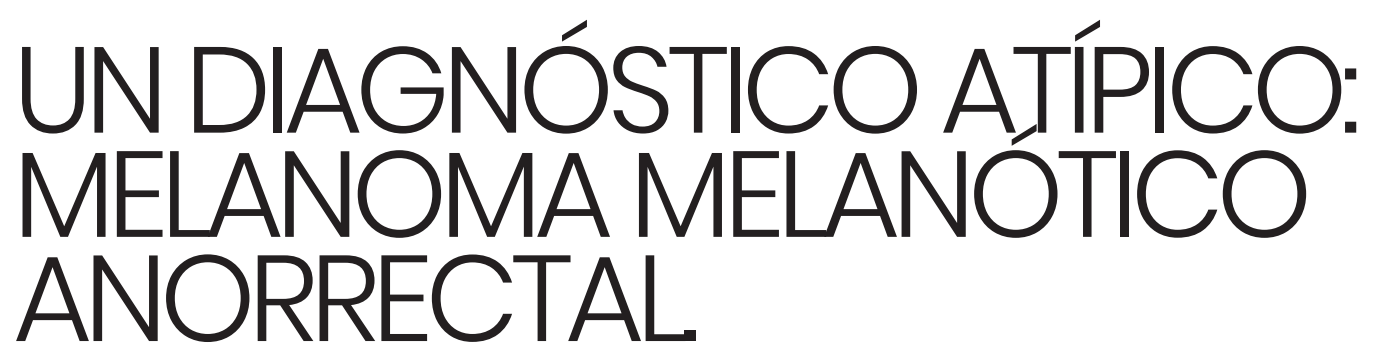

\title{
AN ATYPICAL DIAGNOSIS: MELANOTIC ANORECTAL MELANOMA.
}

\section{Abellán Alfocea P, Salvador Rodríguez L, Martínez Cara JG}

Hospital Universitario Virgen de las Nieves. Granada.

\section{Resumen}

Presentamos el caso de un paciente con melanoma rectal melanocítico, diagnosticado por colonoscopia, durante el estudio de una rectorragia que se había relacionado con patología hemorroidal. El melanoma anorrectal es una entidad muy poco frecuente que representa menos del $1 \%$ de las neoplasias colorrectales y solo un tercio de los casos son melanomas pigmentados. Remitimos esta imagen al ser inusual y representativa de esta patología, ya que el diagnóstico precoz es importante y puede mejorar el pronóstico de estos pacientes.

Palabras clave: melanoma rectal melanocítico, colonoscopia.

\section{CORRESPONDENCIA}

Patricia Abellan Alfocea

Hospital Universitario Virgen de las Nieves

18014 Granada

patriabellan91@gmail.com

Fecha de envío: 24/03/2020

Fecha de aceptación: 07/04/2020

\section{Abstract}

We present the case of a patient with melanocytic rectal melanoma, diagnosed by colonoscopy, during the evaluation of a hemorrhoid-associated rectal bleeding. Anorectal melanoma is a very rare entity which represents less than $1 \%$ of colorectal neoplasms, and only one third of cases are pigmented melanomas. We submit this image since it is unusual and representative of this pathology. We considerer that the case bears significant clinical interest given the importance of an eraly detection of the clinical picture, with a view to establishing treatment without delay, thereby preventing possible complications.

Keywords: melanocytic rectal melanoma, colonoscopy.

\section{Introducción}

El melanoma maligno anorrectal es un trastorno poco frecuente que representa $<2 \%$ de los melanomas y el $1 \%$ de todos los tumores malignos anorrectales y conlleva un pronóstico considerablemente pobre ${ }^{1}$. Es más frecuente en la 6a-7a década, especialmente en mujeres y caucásicos ${ }^{2}$. Las lesiones proliferan 
principalmente en el canal anal y menos frecuente en la línea dentada y el recto. Debido a sus síntomas inespecíficos y su baja incidencia, que conducen a un bajo índice de sospecha, hasta el $40 \%$ de los pacientes presentan enfermedad metastásica al diagnóstico. La resección quirúrgica es el mejor tratamiento, sin embargo, no ha demostrado mejorar la supervivencia. Además, no existe consenso sobre el uso de tratamientos sistémicos ${ }^{1}$.

\section{Caso clínico}

Paciente varón de 60 años, sin antecedentes de interés, que consultó por presentar clínica de proctalgia y rectorragia que se asoció a patología hemorroidal. A la exploración anal destaca la presencia de hemorroides externas y una papila anal hipertrófica pigmentada (Figura 1). Se decidió la realización de sigmoidoscopia detectándose desde el margen anal hasta $7 \mathrm{~cm}$ de este, una lesión mamelonada con mucosa de coloración negruzca y zona central ulcerada, la cual fue biopsiada (Figura 2 y 3 ). En dichas biopsias se detectaron células melánicas malignas confirmando así el diagnóstico de sospecha, melanoma anorrectal. Se realizó estudio de extensión con presencia de metástasis múltiples a distancia que causaron el fallecimiento del paciente en pocos meses.

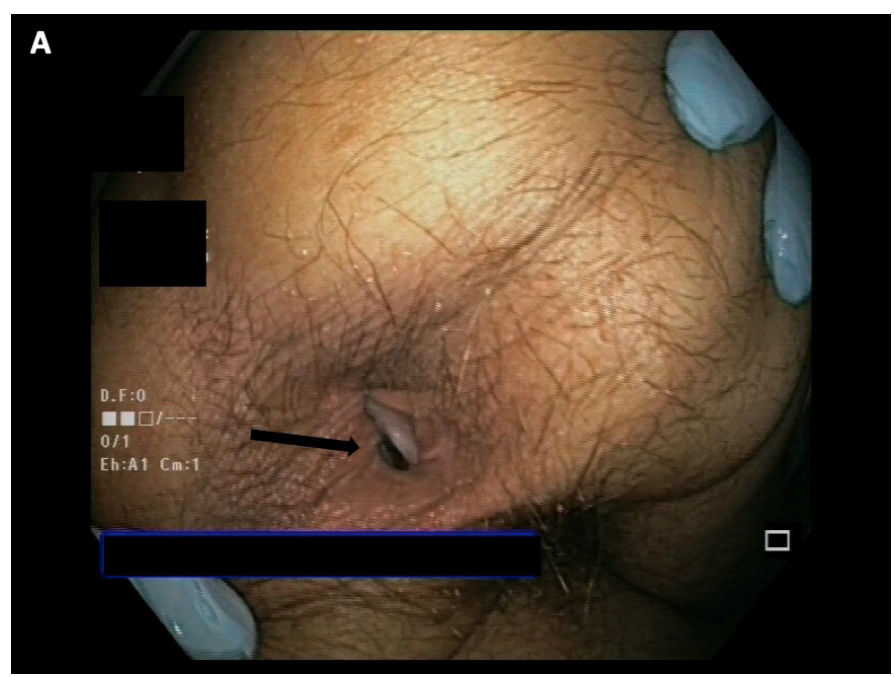

Figura 1

A la inspección anal, se aprecia papila anal pigmentada, sospechosa por su coloración inusual.

\section{Discusión}

La presencia de melanomas en el tracto gastrointestinal es poco habitual, pero a tener en cuenta en el diagnóstico diferencial de neoplasias gastrointestinales por el pronóstico infausto que estos conllevan. Remitimos esta imagen que consideramos de interés al tratarse de una imagen endoscópica poco común y muy característica. En este caso el paciente consultaba por rectorragia que se había asociado a la presencia de patología hemorroidal. Previo a la realización de colonoscopia, se observó la presencia de una lesión protruyente pigmentada a través de canal anal que resultó sospechosa y que refleja la importancia de la exploración física minuciosa antes de establecer diagnósticos clínicos.

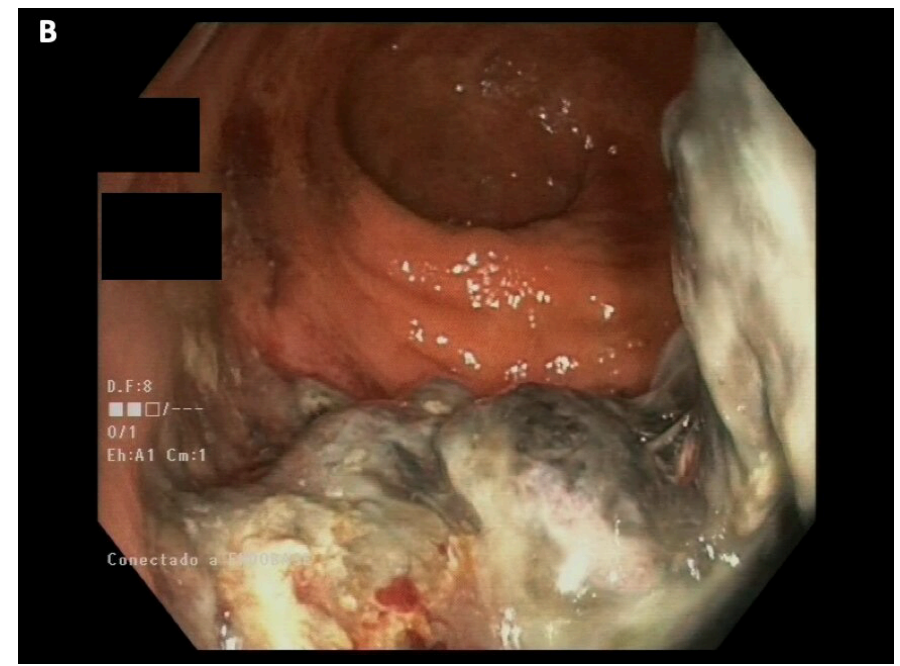

Figura 2

En recto, se aprecia masa mamelonada que ocupa $2 / 3$ de la circunferencia, y donde destaca mucosa muy pigmentada y sin cambios de aspecto adenomatoso.

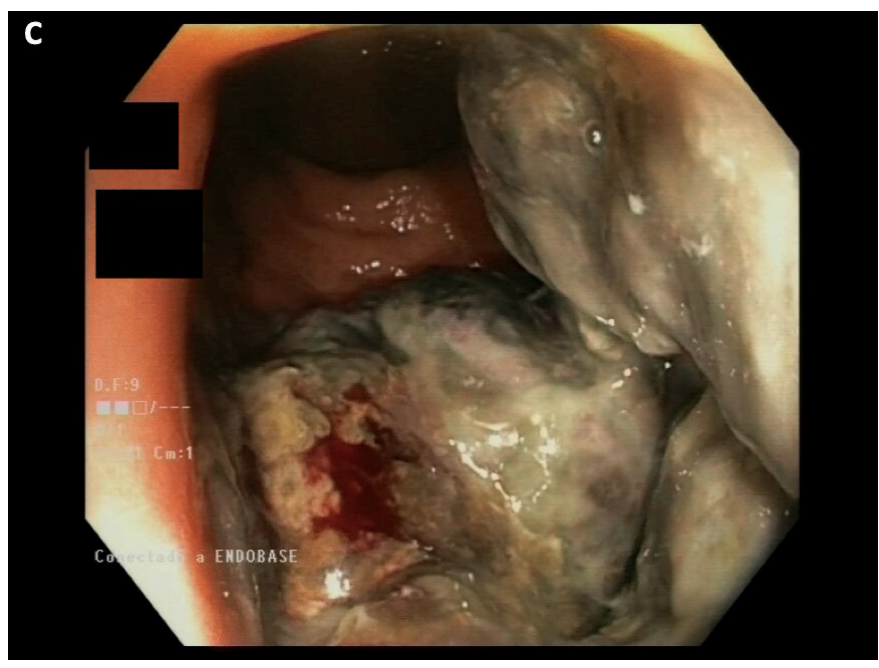

Figura 3

Otra imagen representativa de la lesión, donde apreciamos una zona central ulcerada, friable y cubierta de fibrina.

\section{Bibliografía}

1. Nam S, Kim CW, Baek SJ, et al. The clinical features and optimal treatment of anorectal malignant melanoma. Ann Surg Treat Res. 2014 Sep;87(3):113-7.

2. Serra M, Santos T, Martins $M$ et al. Amelanocytic anorectal malignant melanoma-Case report. Int J Surg Case Rep. 2019;55:164-167 